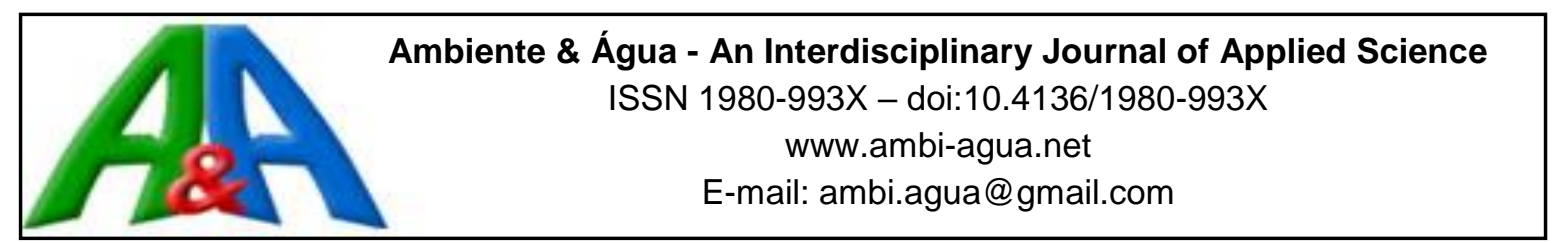

\title{
Potencial de reúso de água na lavagem de caminhões utilizando Contator Biológico Rotativo
}

\author{
doi:10.4136/ambi-agua.1788 \\ Received: 20 Oct. 2015; Accepted: 15 Sep. 2016 \\ Eduardo Lucas Subtil $^{*}$; José Carlos Mierzwa ${ }^{2}$; \\ Ivanildo Hespanhol $^{3}$; Raphael Rodrigues ${ }^{2}$ \\ ${ }^{1}$ Universidade Federal do ABC (UFABC), Santo André, SP, Brasil \\ Centro de Engenharia Modelagem e Ciências Sociais Aplicadas (CECS) \\ ${ }^{2}$ Universidade de São Paulo (USP), São Paulo, SP, Brasil \\ Departamento de Engenharia Hidráulica e Ambiental (PHA) \\ ${ }^{3}$ Universidade de São Paulo (USP), São Paulo, SP, Brasil \\ Centro Internacional de Referência em Reúso de Água (CIRRA) \\ *Autor correspondente: e-mail: eduardo.subtil@ufabc.edu.br, \\ mierzwa@usp.br, ivanhes@usp.br, raphaelrph@gmail.com
}

\section{RESUMO}

Este estudo teve como objetivo avaliar o potencial de reúso de água na lavagem de caminhões a partir do efluente tratado por um Contator Biológico Rotativo operado em escala real. Para avaliação do potencial de reúso foi realizado um balanço de massa para o sistema de reúso levando em consideração a concentração de Sólidos Dissolvidos Totais como contaminante crítico. O sistema de tratamento produziu um efluente com concentrações médias de cor, turbidez, SDT e $\mathrm{DBO}_{5}$ de $45 \pm 14 \mathrm{uC}, 15 \pm 6,0 \mathrm{NTU}, 244 \pm 99 \mathrm{mg} \mathrm{SDT} / \mathrm{L}$ e $14 \pm 7,3 \mathrm{mg} \mathrm{O}_{2} / \mathrm{L}$, respectivamente. Com base no balanço de massa e considerando o valor de SDT estabelecido na NBR 13.696, caso não fosse adotado o enxágue final com água limpa, o potencial de reúso seria de $40 \%$. No entanto, adotando-se $30 \%$ do enxágue final com água limpa, seria possível reutilizar até $70 \%$ do efluente tratado sem comprometer o desempenho da lavagem dos caminhões. A adoção do sistema de reúso possibilitaria uma redução de $\mathrm{R} \$ 2.590,75 / \mathrm{mês}$ no custo operacional do lava-rápido.

Palavras-chave: contator biológico rotativo, lava rápido de caminhão, reúso de água.

\section{Water reuse potential in truck wash using a Rotating Biological Contactor}

\begin{abstract}
This study evaluated the water reuse potential for truck washing using the effluent treated by a Rotating Biological Contactor (RBC) operated in full scale. In order to evaluate the reuse potential, a mass balance was performed for the reuse system taking into account the concentration of Total Dissolved Solids as the critical contaminant. The treatment system produced an effluent with average concentration of color, turbidity, TDS and $\mathrm{BOD}_{5}$ of $45 \pm 14 \mathrm{uC}, 15 \pm 6.0 \mathrm{NTU}, 244 \pm 99 \mathrm{mg}$ TDS / L and $14 \pm 7.3 \mathrm{mg} \mathrm{O}_{2} / \mathrm{L}$, respectively. Based on the mass balance, and considering the TDS concentration established in NBR 13.696, if
\end{abstract}


the final rinse does not use clean water, the potential for effluent reuse can reach $40 \%$. However, if clean water is used as $30 \%$ of the total rinsing volume, it would be possible to reuse $70 \%$ of the treated effluent without compromising truck washing performance. This water reuse approach would result in an operational cost reduction of $\mathrm{R} \$ 2,590.75 /$ month.

Keywords: rotating biological contactor, truck wash, water reuse.

\section{INTRODUÇÃO}

No Brasil, o custo elevado da água industrial, particularmente nas regiões metropolitanas, tem estimulado as indústrias nacionais a ampliar as possibilidades internas de reúso (Hespanhol, 2003). Por exemplo, no estado de São Paulo, o serviço de saneamento (água e esgoto) fornecido pela principal concessionária pode chegar a $\mathrm{R} \$ 34,92$ o metro cúbico (ARSESP, 2016), caso o fator de carga poluidora do efluente seja igual a um. Além dos custos relacionados com os serviços de saneamento, os benefícios inerentes à utilização de água recuperada para usos benéficos incluem a preservação de fontes de qualidade elevada, proteção ambiental e benefícios econômicos e sociais. Dessa forma, a reutilização de água não apenas no setor industrial, mas também no setor de prestação de serviços, é uma abordagem sustentável para adaptação à crescente demanda por água em grandes centros urbanos. (Garcia-Cuerva et al., 2016).

Inserido neste cenário, o serviço de lavagem de veículos apresenta-se como consumidor de grandes volumes de água, podendo se beneficiar do reúso de seus efluentes (Panizza e Cerisola, 2010). O processo padrão para lavagem de carros pode consumir até $200 \mathrm{~L}$ de água por veículo (Boluarte et al., 2016), ao passo que o consumo de água para a lavagem de caminhões pode variar de 350 a 900 litros por veículo, dependendo da tecnologia de lavagem utilizada (Huybrechts et al., 2002). No Brasil, considerando a frota nacional de veículos, estima-se que o volume de água mensal para lavagem de automóveis, ônibus e caminhões seja da ordem de 7 milhões de metros cúbicos por mês (Etchepare, 2012). Além do grande volume, este setor ainda apresenta a vantagem de que o efluente tem melhor aceitação por parte dos usuários pelo fato de não apresentar potencial de contaminação, comparado a esgotos domésticos e certos tipos de efluentes industriais (Lau et al., 2013). Tais características tornam bastante promissora a avaliação de métodos de tratamento para reutilização de água neste setor.

No processo de lavagem de veículos, a sujeira é removida tanto por ação física quanto química. Assim, a composição do efluente gerado pode variar de acordo com o tipo de lavagem e produtos utilizados. De acordo com Hamada e Miyazaki (2004), as principais impurezas presentes neste efluente são areia, poeira, óleo mineral, cera e surfactantes. Outros autores ainda citam a presença de graxas, desengraxantes, sais, carbono e asfalto carreados da superfície do carro (Jönsson e Jönsson, 1995), matéria orgânica e metais (Panizza e Cerisola, 2010).

Em razão do volume e da complexidade do efluente gerado, tanto do ponto de vista de proteção ambiental, como do uso racional dos recursos hídricos, o reúso de água na lavagem de veículos tornou-se assunto relevante em diversos países. Nesse sentido, legislações e diretrizes para uso da água nos processos de lavagem de veículos têm sido estabelecidas em todo o mundo. Como exemplo, pode-se citar que em Queensland, Australia, é obrigatório o uso de, no máximo, 70 litros de água em uma única lavagem de carro, e na Europa, alguns países restringem o consumo de água de 60-70 L por carro e/ou impõe um percentual de recuperação de 70-80\% dá água (QWC, 2008a; 2008b; Boussu et al., 2007). Também na Bélgica, um percentual de reciclagem de $70 \%$ é necessário para que prestadores de serviço de lavagem obtenham a licença ambiental (Huybrechts et al., 2002). Atualmente, quase $15 \%$ dos 
lava-rápidos de carros na Bélgica já tratam o efluente gerado e reutilizam, aproximadamente, $55 \%$ do efluente tratado no processo de lavagem (Boussu et al., 2007).

No cenário nacional, a preocupação com o efluente gerado nesse ramo de atividade associado ao estresse hídrico observado em algumas regiões levou, recentemente, ao desenvolvimento de legislações que abordam essa temática. Vale ressaltar a lei 9.439 de 03/05/2010 do Estado do Espírito Santo que torna obrigatório que os postos de combustíveis, lava-jatos, transportadoras, empresas de ônibus e locadoras de veículos, que mantêm pontos de lavagem, higienização e desengraxamento ou congêneres, instalem o sistema de tratamento e reutilização de água (Espírito Santo, 2010). Leis semelhantes a esta foram sancionadas nos estados do Rio de Janeiro em 08 de setembro de 2011 (Lei n6.034, Rio de Janeiro, 2011), de Goiás, em 18 de agosto de 2010, modificada em 08 de março de 2012 (Lei n ${ }^{\circ} 17.128$ e Lei ${ }^{\circ}$ 17.582, Goiás, 2010; 2012) e na cidade de São Paulo, em 13 de abril de 2015 (Lei nº 16.160, São Paulo, 2015).

Tendo em vista a tendência em todo o país para que a prática do reúso possa ser adotada na atividade de lavagem de veículos, é importante o planejamento de toda a estrutura para o reúso. Além disso, devem ser aplicadas técnicas de tratamento que sejam adequadas ao efluente gerado e que ocupem pouco espaço, devido à pequena disponibilidade de área nas estações de lavagem de veículos. (Boluarte et al., 2016). Da mesma forma, é necessário que o sistema de reúso proposto apresente viabilidade econômica. Algumas tecnologias que foram propostas e testadas incluem separação por membranas de Ultrafiltração (UF) (Jonsson e Jonsson, 1995; Hamada e Miyazaki, 2004), Osmose Reversa e Nanofiltração (NF) (Brown, 2000; Boussu et al., 2007, Lau et al., 2013); oxidação eletroquímica (Panizza e Cerisola, 2010) e tratamento físico-químico (Bhatti et al., 2010; Zaneti et al., 2011). Ainda que algumas pesquisas tenham sido feitas e distintas tecnologias testadas, os critérios de qualidade da água para lavagem de veículos não são bem estabelecidos (Zaneti et al., 2011).

A prática de reúso interno em qualquer processo pode resultar no aumento da concentração de determinados compostos que não são removidos pelo processo de tratamento adotado (build-up), o que pode limitar o potencial de reutilização de água (Zanetti et al., 2012). De maneira geral, a concentração de Sólidos Dissolvidos Totais (SDT) é utilizada como parâmetro crítico para se estabelecer o potencial de reúso, já que este é um parâmetro restritivo para o uso da água em diversas aplicações industriais, incluindo a lavagem de veículos. Concentrações elevadas de SDT podem deixar manchas no veículo e, dependendo da concentração, podem acelerar o processo de corrosão. Considerando esse efeito de acumulação, sistemas de tratamento biológicos apresentam grande potencial para a prática de reúso de água por não promoverem um aumento na carga de sólidos dissolvidos, quando comparados a sistemas físico-químicos.

Um sistema biológico com notórios resultados no tratamento de efluentes com foco em reúso é o Contator Biológico Rotativo (CBR). Este sistema tem sido utilizado com sucesso para o tratamento de diferentes efluentes, incluindo esgoto tipicamente doméstico (Akunna e Jefferies, 2000; Griffin e Findlay, 2000; Nowak, 2000), efluentes de aquicultura (Brazil, 2006) e águas cinzas (May, 2009). O CBR fornece condições ideais para crescimento de micro-organismos em um biofilme estático, o que permite a aplicação de maiores cargas orgânicas e uma maior resistência a choques tóxicos, quando comparado a métodos de culturas suspensas (Hassard et al., 2015). O CBR apresenta simplicidade e menor custo operacional em comparação ao sistema de lodos ativados convencional. Considerando estas vantagens, este estudo teve por objetivo avaliar o potencial de reúso de água, a partir do efluente tratado por um processo biológico aeróbio do tipo Contator Biológico Rotativo (CBR) operado em escala real, levando em consideração a concentração de Sólidos Dissolvidos Totais como parâmetro crítico. 


\section{MATERIAIS E MÉTODOS}

\subsection{Sistema de tratamento de efluentes}

O presente estudo foi realizado com base no sistema de tratamento de efluentes instalado em um Lava Rápido de Caminhões, localizado no km 24,2 da Rodovia Anhanguera, sentido Capital-Interior, no Estado de São Paulo. O sistema de tratamento era composto por uma unidade de desarenação e separação de óleo e água, tanque de equalização $\left(8,6 \mathrm{~m}^{3}\right)$, sistema de tratamento biológico aeróbio CBR com discos em material polimérico, seguido por filtro de alta taxa composto por areia e antracito (área de filtração de $0,073 \mathrm{~m}^{2}$ ), sendo o efluente clorado ao final do processo. Com exceção do filtro de areia e do sistema de cloração, as unidades de tratamento foram construídas em alvenaria. A estação de tratamento operou continuamente com uma vazão média de $300 \mathrm{~L} / \mathrm{h}$, resultando em um volume médio diário de efluente tratado de $7,2 \mathrm{~m}^{3}$.

O dimensionamento do sistema CBR foi realizado com base em dados de literatura, de forma a evitar que a carga orgânica superficial ultrapassasse o valor de $32 \mathrm{~g}$ de $\mathrm{DBO}_{5} / \mathrm{m}^{2}$ dia (Grady et al., 1999) e uma $\mathrm{DBO}_{5}$ média do efluente de $800 \mathrm{mg} \mathrm{O}_{2} / \mathrm{L}$, obtida por meio de quatro medidas mensais prévias da DQO do efluente gerado $(2.500 \mathrm{mg} / \mathrm{L})$, adotando-se um fator de correlação $\mathrm{DQO} \mathrm{DBO}_{5}$ igual a 3 , e vazão diária de efluente de $8 \mathrm{~m}^{3}$, resultando em 180 discos com uma área útil total de $203 \mathrm{~m}^{2}$.

O sistema de CBR foi projetado com 4 câmaras interligadas. Cada câmara continha 45 discos, equiespaçados em $2 \mathrm{~cm}$. Cada disco possuía área de $1,13 \mathrm{~m}^{2}$, resultando em uma área útil de tratamento de $203 \mathrm{~m}^{2}$. A tubulação de saída do efluente estava localizada próxima à altura do eixo dos discos, de modo a assegurar a aeração do biofilme aderido através do contato direto com o ar. O volume total do CBR era de $3,2 \mathrm{~m}^{3}$, o que resultava em um Tempo de Detenção Hidráulica médio de 10,5 horas.

A dosagem de nutrientes no reator foi prevista de maneira a assegurar a relação Carbono:Nitrogênio:Fósforo de 100:5:1, ideal ao processo de degradação biológica. Ureia foi utilizada como fonte de nitrogênio, e tripolifosfato de sódio foi utilizado como fonte de fósforo. Também foram previstos um sistema de dosagem de hidróxido de sódio para ajuste do $\mathrm{pH}$ do afluente ao sistema CBR e um sistema de dosagem de hipoclorito de sódio para a desinfecção final do efluente. A rotação dos discos do sistema CBR foi mantida em 2 rpm, através de conjunto moto-redutor. A Figura 1 ilustra o arranjo do sistema de tratamento de efluentes do lava-rápido.

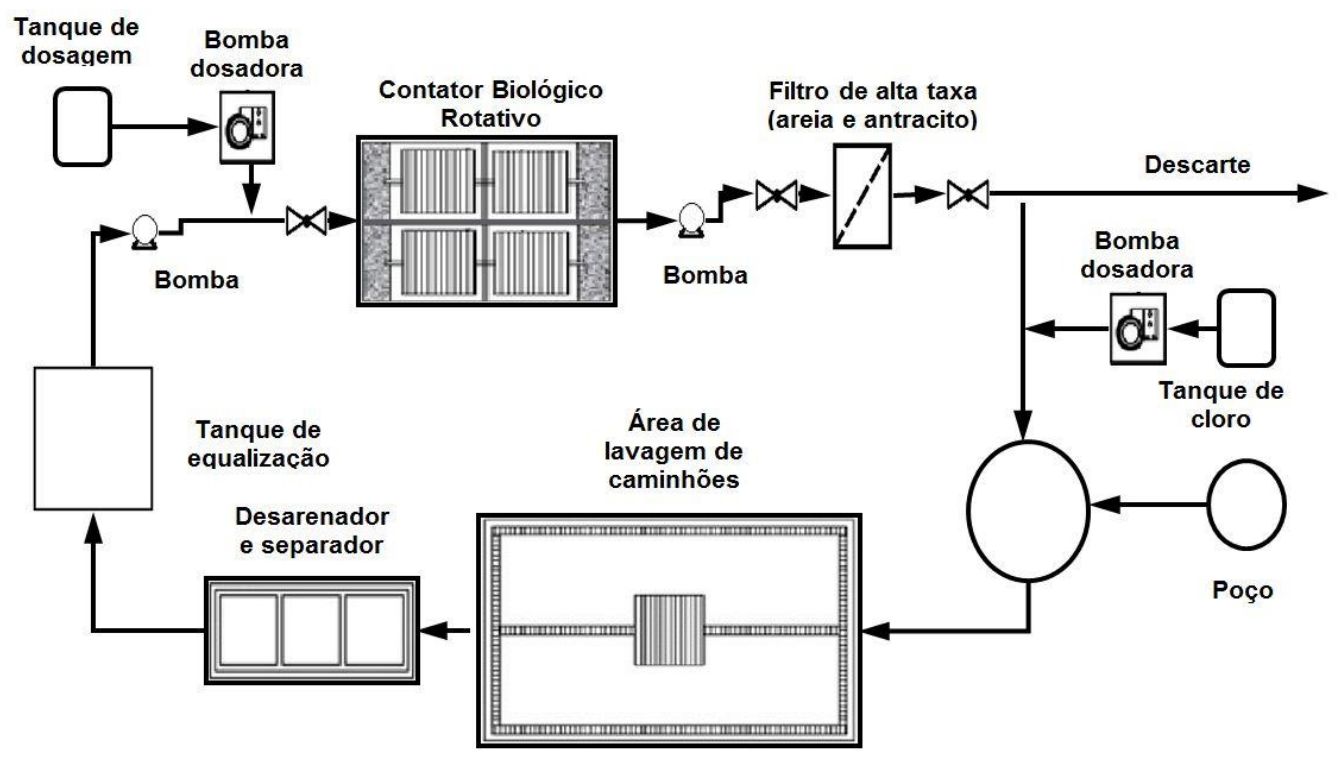

Figura 1. Fluxograma da Estação de Tratamento de Efluentes. 


\subsection{Monitoramento do sistema de tratamento}

Após atingir condições estáveis de operação, o sistema de tratamento foi monitorado durante um período de 5 meses. Ao todo foram realizadas 18 coletas com frequência semanal. As amostras foram coletadas do tanque de equalização, após o reator biológico (CBR) e após o sistema de filtração (água que seria reutilizada). As variáveis de qualidade monitoradas em cada ponto amostral conforme descrito anteriormente estão apresentadas na Tabela 1.

Tabela 1. Variáveis monitoradas no sistema de tratamento e os métodos utilizados.

\begin{tabular}{ll}
\hline \multicolumn{1}{c}{ Parâmetro } & \multicolumn{1}{c}{ Método } \\
\hline $\mathrm{pH}$ & Método eletrométrico- pH-metro (Quimis) \\
$\mathrm{Cor}$ & Espectrofotometria visível - Colorímetro (Policontrol) \\
Turbidez & Nefelométrico - Turbidímetro (Policontrol) \\
Condutividade & Analisador multi-parâmetro (Hexis) \\
Sólidos Dissolvidos Totais & Analisador multi-parâmetro (Hexis) \\
DBO 5 & Respirométrico - aparelho OxiDirect (Aqualitic) \\
DQO & Oxidação por dicromato de potássio- 5210 B Standard Methods* \\
COT & Analisador TOC-V CPH (Shimadzu) \\
\hline
\end{tabular}

*APHA (2005).

\subsection{Estimativa do potencial de reúso}

A eficiência do sistema para a remoção de contaminantes específicos foi levada em consideração para a determinação do potencial de reúso. Contaminantes que não foram removidos pelo sistema de tratamento de efluentes foram considerados críticos. Considerando a utilização de um sistema biológico de tratamento, foram definidos como contaminantes críticos os sais inorgânicos, cuja concentração pode ser indiretamente avaliada pela determinação de Sólidos Dissolvidos Totais (SDT). A opção pelos SDT como variável de controle para a determinação do potencial de reúso aplica-se na água utilizada em diversos processos industriais, incluindo a lavagem de veículos, pois sua concentração é restringida e sua remoção é limitada (Hespanhol e Mierzwa, 2005).

De acordo com a NBR 13.969 de 1997 (ABNT, 1997), na lavagem de veículos a concentração máxima de SDT na água de reúso deve ser de até a $200 \mathrm{mg} / \mathrm{L}$. Considerando este limite foi possível determinar, por meio de balanço de massa, o aporte destes contaminantes no processo de lavagem tendo como base as concentrações de SDT na água disponível para lavagem e no efluente resultante. Após a inclusão da linha de reúso no fluxograma do processo, foi possível estimar o potencial máximo de reúso de efluentes deste sistema (Figura 2).

Para possibilitar a operação do sistema de lavagem com água de reúso com uma concentração de SDT dentro dos limites e minimizar a ocorrência de efeitos adversos na operação de lavagem, foi estabelecido um percentual mínimo de utilização de água limpa para a realização do enxágue final do veículo, o que foi considerado na avaliação apresentada.

Com base nestas considerações, o potencial de reúso foi avaliado a partir dos resultados de análises das amostras de água e efluentes coletados antes e depois do sistema de tratamento por CBR e filtração, além da caracterização da água limpa utilizada no processo de lavagem. Para o cálculo do potencial de reúso determinou-se, primeiramente, a carga de SDT incorporada no sistema durante o processo de lavagem, para posteriormente avaliar a variação de sua concentração em função do percentual de reúso adotado. Ressalta-se que nesta fase do 
estudo, o efluente tratado não era reutilizado. A seguir são apresentadas as Equações (1 a 8), resultantes do balanço de massa para estimativa do potencial de reúso. Vale ressaltar que as perdas por evaporação e por vazamento foram consideradas desprezíveis.

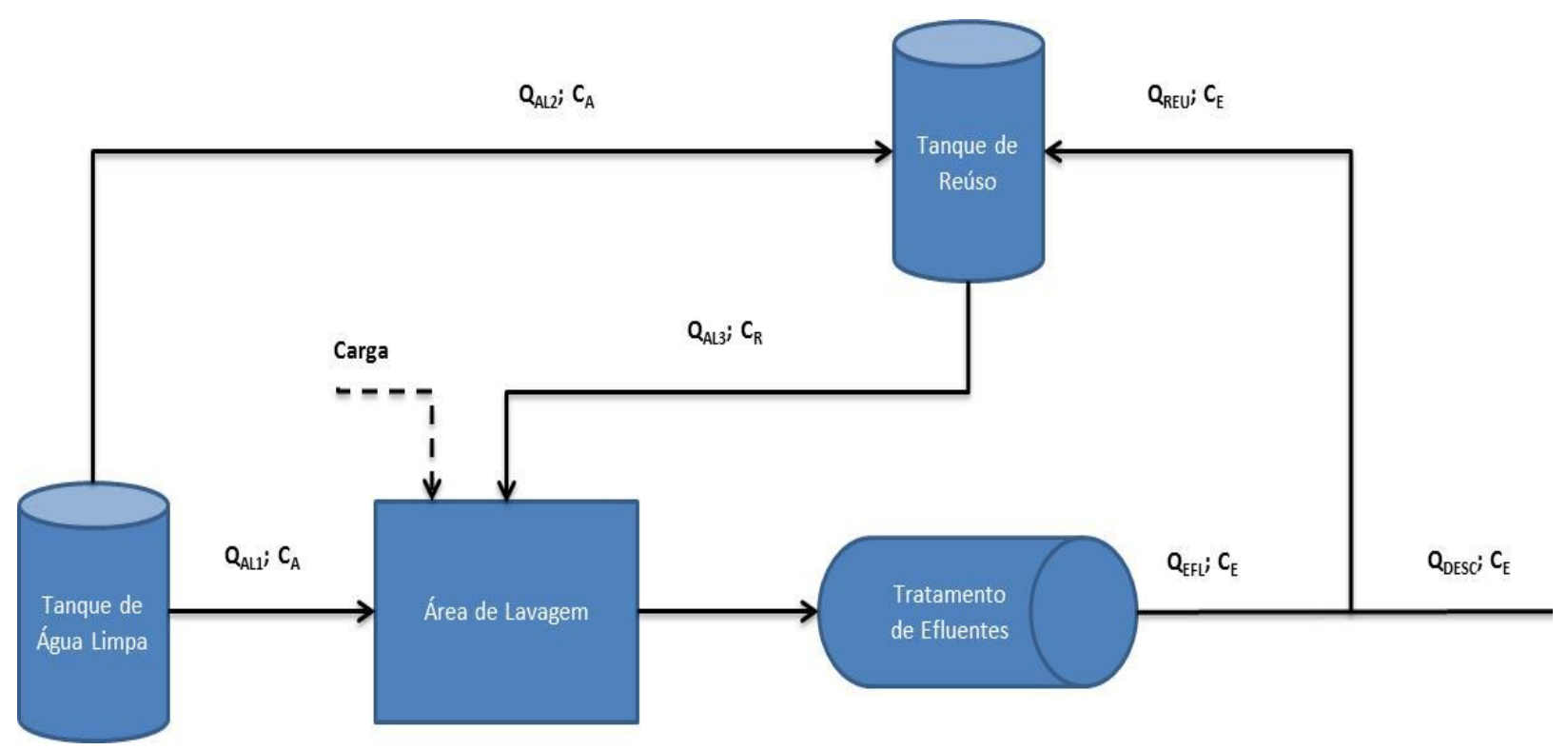

Figura 2. Diagrama de correntes consideradas para o sistema de reúso

$$
\begin{aligned}
& \text { Carga }=Q_{E F L} \cdot C_{E}-Q_{A L} \cdot C_{A} \quad\left(Q_{A L 3}=0\right) \\
& C_{E}=\frac{\text { Carga }}{Q_{\text {Lavagem }}\left\{1-\left[\% R \cdot\left(1-\% L_{A L}\right)\right]\right\}}+C_{A} \\
& C_{R E U}=C_{A}(1-\% R)+C_{E} \cdot \% R \\
& Q_{A L 1}=Q_{\text {Lavagem }} \% L_{A L} \\
& Q_{R E U}=Q_{\text {Lavagem }} \% R \cdot\left(1-\% L_{A L}\right) \\
& Q_{A L 2}=Q_{\text {Lavagem }} \cdot\left(1-\% L_{A L}\right) \cdot(1-\% R) \\
& Q_{D E S C}=Q_{A L 1}+Q_{A L 2} \\
& \% R=\frac{Q_{R E U}}{\left(Q_{\text {lavagem }} \cdot\left(1-\% L_{A L}\right)\right.} \cdot 100
\end{aligned}
$$

em que:

QLavagem: vazão de lavagem de caminhões ( $\left.\mathrm{m}^{3} / \mathrm{dia}\right)$;

Carga: carga de SDT incorporada no sistema (g/dia);

$\mathrm{C}_{\mathrm{REU}}$ : concentração de SDT na água de reúso (mg/L);

$\mathrm{C}_{\mathrm{A}}$ : concentração de SDT na água limpa $(\mathrm{mg} / \mathrm{L})$;

$\mathrm{C}_{\mathrm{E}}$ : concentração de SDT no efluente $(\mathrm{mg} / \mathrm{L})$; 
$\mathrm{Q}_{\mathrm{AL}}$ : vazão de água limpa $\left(\% \mathrm{R}=0\right.$ ou $\left.\% \mathrm{~L}_{\mathrm{AL}}>0\right)\left(\mathrm{m}^{3} / \mathrm{dia}\right)$;

QDESC: vazão de efluente descartada $\left(\mathrm{m}^{3} / \mathrm{dia}\right)$; $\left(\mathrm{m}^{3} / \mathrm{dia}\right)$;

$\mathrm{Q}_{\mathrm{AL2}}$ : vazão de água limpa misturada ao efluente, para repor o descarte de efluente

$\mathrm{Q}_{\text {AL3}}$ : vazão de água de lavagem antes do enxágue final( $\left.\mathrm{m}^{3} / \mathrm{dia}\right)$;

$\% \mathrm{~L}_{\mathrm{AL}}$ : porcentagem de água limpa no enxágue final, e

$\% \mathrm{R}$ : porcentagem de recirculação de efluente.

Um aspecto a ser considerado na estrutura de reúso proposta está relacionado ao tempo necessário para que a água de reúso atinja a concentração limite do contaminante de controle. O tempo depende do volume do tanque de armazenagem da água de reúso, da vazão de água limpa e do efluente que deixa o sistema. Considerando-se o diagrama apresentado na Figura 2, é possível obter uma equação que correlaciona a variação da concentração do contaminante de controle na água de reúso em função do tempo e do volume do tanque de reúso (Equação 9). Para a obtenção da equação 9 foi considerada a mistura imediata e completa entre a água limpa e efluente no tanque de reúso.

$$
C_{(t)}=C_{A}-\frac{\operatorname{Carga}}{Q_{\text {Desc }}} \cdot\left(e^{-\frac{t}{\theta}}-1\right)
$$

em que: $(\mathrm{mg} / \mathrm{L})$

$\mathrm{C}_{(\mathrm{t})}=$ concentração do contaminante de controle no tanque de reúso em função do tempo (dias);

$\theta=$ tempo de detenção hidráulico no tanque de reúso (Volume do tanque $\left(\mathrm{m}^{3}\right) / \mathrm{Q}_{\text {desc }}$ )

$\mathrm{t}=$ tempo de operação do sistema de lavagem (dias).

A partir dos resultados obtidos no monitoramento do sistema foi possível estimar o potencial de reúso de efluentes e o tempo necessário para que a concentração do contaminante de controle atingisse um valor estável em função do volume do tanque de reúso.

A avaliação da redução nos custos de operação foi feita com base nos dados sobre o potencial de redução do consumo de água no lava-rápido e considerando a captação de água limpa de um poço semi-artesiano com 50 metros de profundidade. O cálculo da potência da bomba de captação do poço foi feito com base na expressão apresentada em Porto (2006), admitindo-se uma eficiência do conjunto moto-bomba de 50\%. A estimativa na redução do custo da destinação dos efluentes foi obtida considerando-se as tarifas médias praticadas pela companhia de saneamento local (ARSESP, 2016), admitindo-se uma economia comercial e a tarifa média de energia praticada pela concessionária na Região Metropolitana de São Paulo (ANEEL, 2016), que é de R $\$ 0,54346 / \mathrm{kWh}$.

\section{RESULTADOS E DISCUSSÃO}

\subsection{Característica do efluente gerado}

Em termos quantitativos, o sistema de lavagem de caminhão utilizado como estudo produzia uma média de $7.200 \mathrm{~L} /$ dia de efluente, resultante da lavagem diária de 16 caminhões, correspondendo a um consumo médio de 450 litros por caminhão. Este valor encontra-se dentro da faixa de variação de 300 a 900 litros citado por Huybrechts et al. 
(2002). Comparando esse dado com os valores médios de lava-rápidos de carros de passeio fica evidente a diferença no consumo de água, uma vez que Boluarte et al (2016) estimam um consumo de 200L por lavagem e Zaneti et al. (2011) estimam um consumo de $120 \mathrm{~L}$ por veículo lavado. Além da diferença em relação ao tipo de veículo lavado, a utilização ou não de equipamento de lavagem apresenta grande influência na quantidade de água consumida. Brown (2002) mostrou uma diferença no consumo de água maior que $500 \%$ quando comparou uma lavagem por túnel $(268 \mathrm{~L})$ com uma manual $(45 \mathrm{~L})$.

As características da água utilizada no lava-rápido de caminhão e do efluente gerado no processo de lavagem após o tratamento preliminar (remoção de areia e óleo), ou seja, antes do tratamento biológico, encontram-se apresentadas na Tabela 2. Os valores de matéria orgânica expressos pela DQO $\left(626 \pm 125 \mathrm{mg} \mathrm{O}_{2} \mathrm{~L}^{-1}\right)$ e $\mathrm{DBO}_{5}\left(169 \pm 24 \mathrm{mg} \mathrm{O}_{2} \mathrm{~L}^{-1}\right)$, estão dentro da faixa de valores para esgoto sanitário tipicamente doméstico, onde a concentração de DQO varia, geralmente, de 800 a $200 \mathrm{mg} \mathrm{O} \mathrm{L}^{-1}$ e os de $\mathrm{DBO}_{5}$, de 400 a $100 \mathrm{mg} \mathrm{O}_{2} \mathrm{~L}^{-1}$ (Jordão e Pessoa, 2011). Destaca-se que o valor obtido para DQO está abaixo daquele utilizado para o dimensionamento do CBR, indicando a possibilidade de otimização do projeto. Em comparação a efluentes de lava-rápidos de veículos leves, os valores de DQO e $\mathrm{DBO}_{5}$ obtidos neste estudo são significativamente superiores. Zaneti et al. (2011) reportaram valores de DQO e $\mathrm{DBO}_{5}$, respectivamente, de $241 \pm 23,5$ e $133 \pm 61 \mathrm{mg} \mathrm{O}_{2} \mathrm{~L}^{-1}$ para o primeiro experimento e $191 \pm 22$ e $68 \pm 13 \mathrm{mg} \mathrm{O}_{2} \mathrm{~L}^{-1}$ para o segundo experimento, enquanto que Boussu et al. (2007) reportaram concentrações de $\mathrm{DBO}_{5}$ e DQO variando entre 95 a $140 \mathrm{mg}$ $\mathrm{O}_{2} \mathrm{~L}^{-1}$ e 208 a $382 \mathrm{O}_{2} \mathrm{~L}^{-1}$, respectivamente. $\mathrm{O}$ mesmo autor ressalta que os detergentes são os principais responsáveis para a demanda de oxigênio no efluente gerado no processo de lavagem de veículos.

Tabela 2. Características da água limpa utilizada e do efluente gerado na lavagem de caminhão após o tratamento preliminar (separador água e óleo).

\begin{tabular}{llll}
\hline \multicolumn{1}{c}{ Parâmetro } & Água Limpa' & Efluente" & Unidade \\
\hline $\mathrm{pH}$ & $6,2 \pm 0,2$ & $6,1 \pm 0,4$ & -- \\
Cor & $1,6 \pm 0,6$ & $242 \pm 81$ & $\mathrm{uC}$ \\
Turbidez & $0,78 \pm 0,1$ & $156 \pm 45$ & $\mathrm{NTU}$ \\
Condutividade & $92 \pm 13$ & $596 \pm 155$ & $\mu \mathrm{s} / \mathrm{cm}$ \\
SDT & $43 \pm 7$ & $284 \pm 76$ & $\mathrm{mg} \mathrm{SDT} / \mathrm{L}$ \\
DQO & -- & $626 \pm 125$ & $\mathrm{mg} \mathrm{O} / \mathrm{L}$ \\
DBO & $1,0 \pm 1,0$ & $169 \pm 24$ & $\mathrm{mg} \mathrm{O} / \mathrm{L}$ \\
COT & $1,4 \pm 0,3$ & $44 \pm 19$ & $\mathrm{mg} \mathrm{C} / \mathrm{L}$ \\
\hline
\end{tabular}

Legenda: ${ }^{\mathrm{I}}$ Número de amostras $=3$.

${ }^{\text {II }}$ Número de amostras $=18$.

Os valores de matéria orgânica desse estudo estam acima dos valores encontrados por Zaneti et al. (2011) e Boussu et al. (2007). Essa diferença pode ser explicada pelo tipo de veículo lavado (que neste estudo foram caminhões), incluindo o compartimento de carga. Nos referidos estudos, os veículos eram predominante de passeio.

Além da matéria orgânica, pode-se observar pela Tabela 2 que o efluente gerado apresentou elevada concentração de cor e turbidez, atingindo valores médios de $242 \pm 57$ uC e $156 \pm 45$ NTU, respectivamente. Também vale ressaltar a elevação na concentração de SDT quando comparado com a água limpa utilizada no processo de lavagem. O aumento observado 
foi de, aproximadamente, $700 \%$ na concentração de SDT, resultando numa concentração média de $284 \pm 86 \mathrm{mg} \mathrm{L}^{-1}$, que consiste na carga de contaminantes incorporada no processo de lavagem.

\subsection{Desempenho do sistema CBR}

A variação temporal de cor, turbidez, $\mathrm{DBO}_{5}$ e DQO para o afluente e efluente do CBR e do efluente após o sistema de filtração é apresentada na Figura 3. O sistema CBR promoveu uma redução média de $79 \%$ (Figura 4) tanto para cor como para turbidez, resultando num efluente tratado com $51 \pm 16$ uC e $28 \pm 15$ NTU. Além disso, pode-se observar que mesmo com a variação na qualidade do afluente, o CBR produzia um efluente com qualidade relativamente constante em relação a essas variáveis de qualidade. $O$ filtro de alta taxa de areia e antracito utilizado após o tratamento biológico permitiu uma redução adicional média de $16 \%$ para cor e de $46 \%$ para a turbidez (Figura 4), sendo o efluente produzido para reúso com $43 \pm 16 \mathrm{uC}$ e $15 \pm 5$ NTU. Mesmo que a turbidez obtida tenha sido superior ao recomendado pela NBR13.696, ela não pode ser um impeditivo para a reutilização do efluente na operação de lavagem, principalmente nas etapas iniciais, cujo objetivo é remover a sujidade grosseira presente na parte inferior dos caminhões. A remoção de cor e turbidez é importante para a aplicação do reúso, uma vez que o aspecto estético da água é um fator relevante para a sua aceitação. Zaneti et al. (2011), avaliando um sistema físico-químico por flotação por ar dissolvido no tratamento de efluentes da lavagem de veículos visando o reúso, reportaram valores de turbidez de $12 \pm 8 \mathrm{NTU}$, similar ao obtido pelo sistema CBR seguido por filtração deste estudo. Por outro lado, Hamada e Miyazaki, (2004) empregaram a floculação seguida por um sistema de ultrafiltração, atingindo valores de turbidez menores que $0,05 \mathrm{NTU}$, ressaltando-se que os processos propostos por ambos os grupos de pesquisa apresentam limitação para a remoção de matéria orgânica, o que não ocorre com o processo proposto neste estudo.

Ressalta-se que, no caso da utilização de sistemas físico-químicos como o proposto por Zaneti et al. (2011), a utilização de produtos químicos irá resultar na elevação da concentração de SDT, o que conduzirá a um menor potencial de reúso. A mesma observação vale para o processo de tratamento proposto por Hamada e Miyazaki (2004), mesmo com a menor dosagem de produtos químicos utilizada, o que irá contribuir para o aumento no fator de concentração de contaminantes, inclusive dos compostos orgânicos.

Durante o monitoramento do sistema CBR, ocorreu remoção significativa de matéria orgânica em termos de $\mathrm{DBO}_{5}$, atingindo uma eficiência média de $86 \%$, sendo a concentração remanescente no efluente do CBR de $24 \pm 15 \mathrm{mg} \mathrm{O} \mathrm{O}_{2} \cdot \mathrm{L}^{-1}$. Em contrapartida, a redução de DQO e COT foi menos acentuada, com remoções médias de $53 \%$ e $52 \%$, respectivamente, indicando elevada presença de material orgânico recalcitrante. Destaca-se que a relação $\mathrm{DQO}_{/} \mathrm{DBO}_{5}$ variou de aproximadamente 3 no afluente para 12 no efluente do CBR, demonstrando remoção efetiva de matéria orgânica biodegradável. A relação $\mathrm{DQO} / \mathrm{DBO}_{5}$ do processo físico-químico avaliados por Zaneti et al. (2011) apresentaram aproximadamente a mesma relação para o afluente $(2,8)$ e efluente $(2,6)$.

Cabe destacar, como se verifica na Figura 3, que o sistema CBR, mesmo operando com variações significativas na qualidade do afluente, possibilitou a obtenção de um efluente tratado com qualidade relativamente estável, demonstrando a robustez deste sistema em relação aos processos físico-químicos, que irão requerer ajustes nas dosagens químicas e condições operacionais.

$\mathrm{Na}$ Tabela 3 é apresentado um resumo dos dados de qualidade do efluente antes e depois do tratamento pelo sistema avaliado neste estudo. Considerando-se que neste estudo foi estabelecida a concentração de SDT como limitante para a definição do potencial de reúso, é importante destacar o fato da concentração desta variável não ter sofrido alteração 
significativa com a utilização do sistema biológico de tratamento, o que é uma vantagem em comparação às propostas que empregam os sistemas físico-químicos para tratamento do efluente, conforme já destacado. Esta condição resultará em um maior potencial de reúso do efluente.
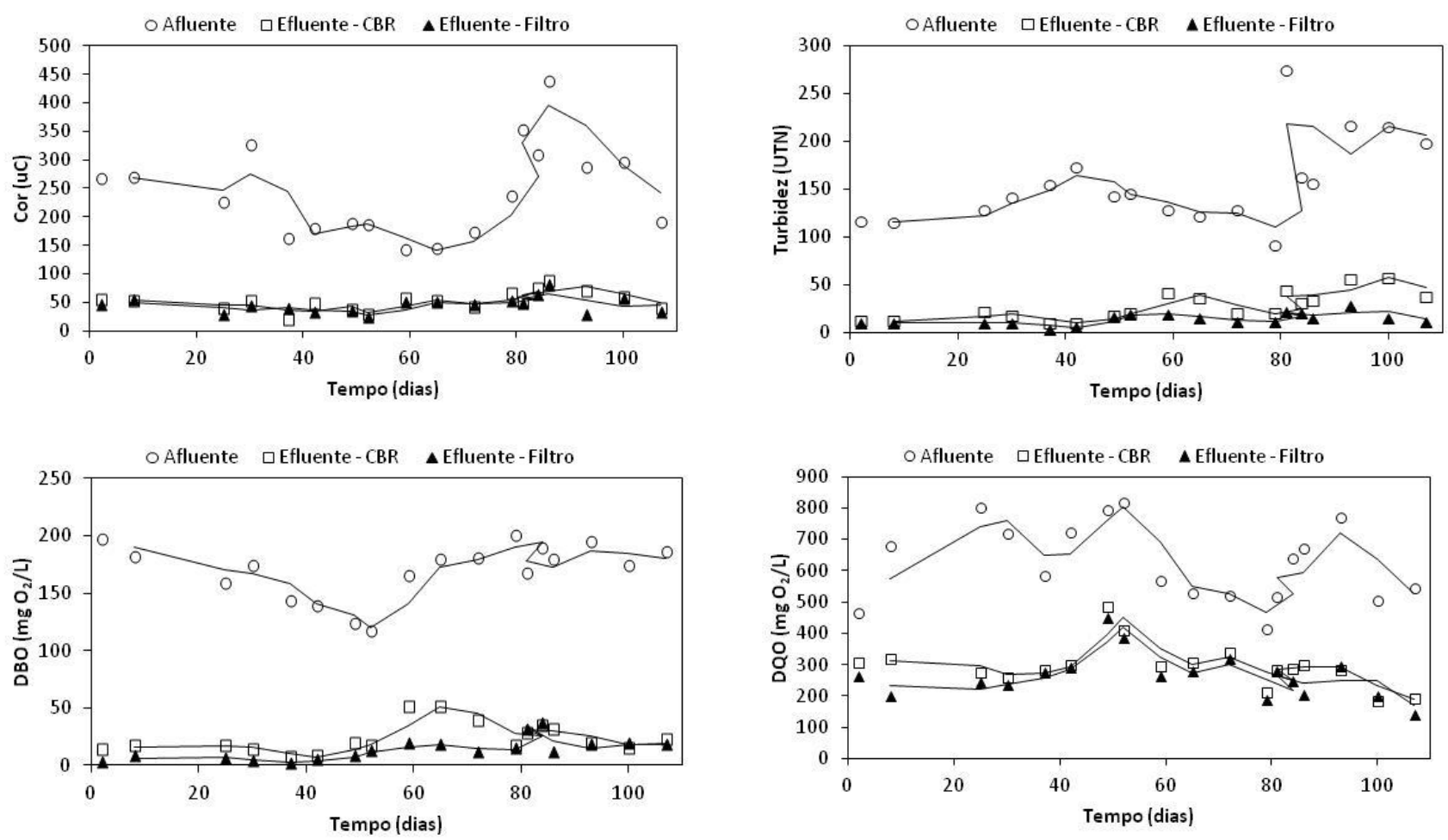

Figura 3. Variação temporal de cor, turbidez, DBO e DQO durante condições estáveis de funcionamento, sendo a linha contínua nos gráficos a média móvel dos dados.

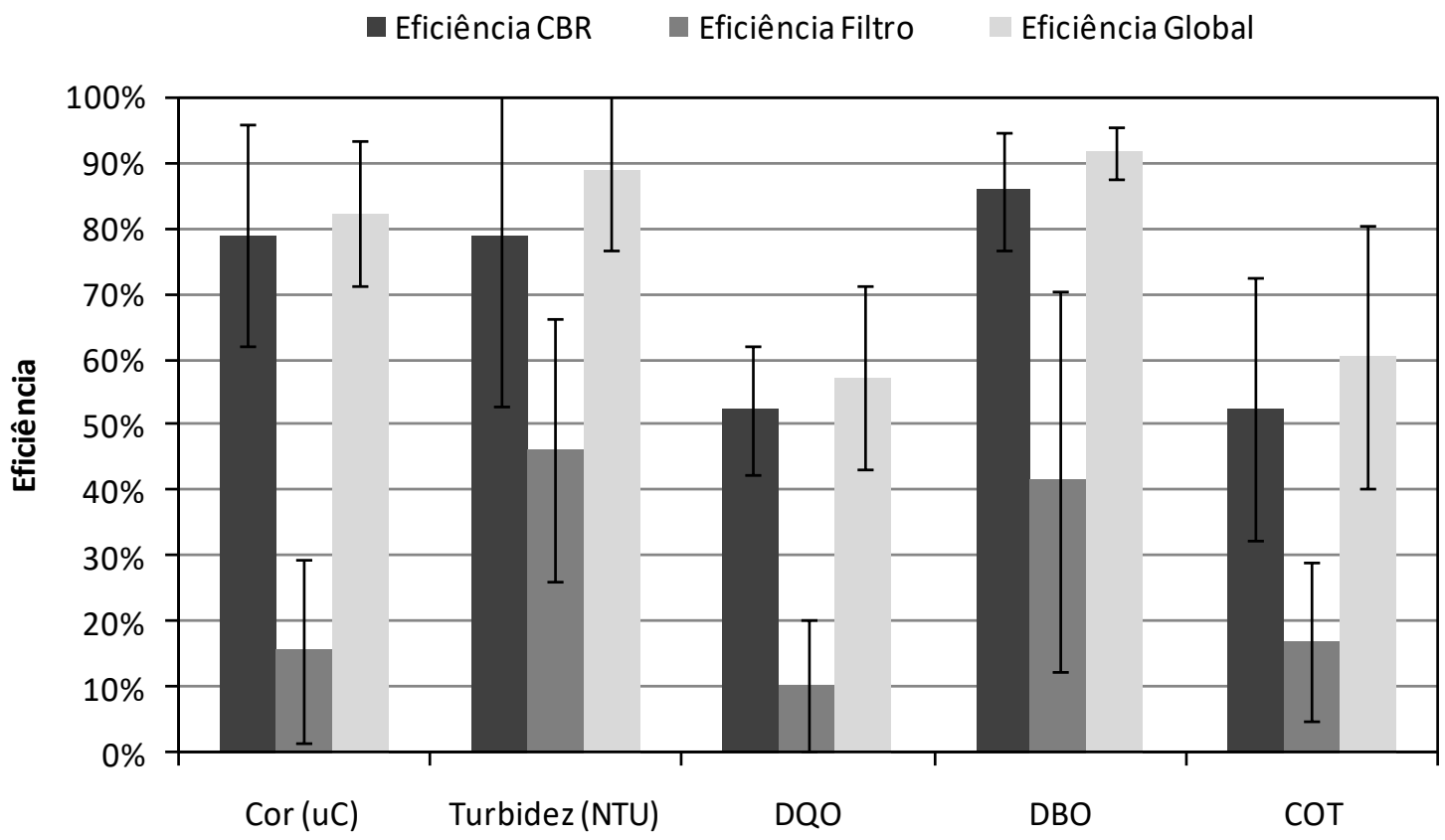

Figura 4. Eficiência do CBR, do sistema de filtração e a eficiência global do sistema. 
Tabela 3. Qualidade do efluente tratado pelo sistema CBR e após o sistema de filtração.

\begin{tabular}{lcccc}
\hline \multicolumn{1}{c}{ Parâmetro } & Antes CBR & Após CBR & Após Filtro & Unidade \\
\hline $\mathrm{pH}$ & $6,1 \pm 0,4$ & $6,0 \pm 0,6$ & $6,0 \pm 0,4$ & -- \\
Cor & $242 \pm 81$ & $51 \pm 16$ & $45 \pm 14$ & $\mathrm{uC}$ \\
Turbidez & $156 \pm 45$ & $28 \pm 15$ & $15 \pm 6,0$ & $\mathrm{NTU}$ \\
Condutividade elétrica & $596 \pm 155$ & $489 \pm 202$ & $518 \pm 214$ & $\mathrm{\mu s} / \mathrm{cm}$ \\
SDT & $284 \pm 76$ & $230 \pm 96$ & $244 \pm 99$ & $\mathrm{mg} \mathrm{SDT} / \mathrm{L}$ \\
DQO & $626 \pm 125$ & $296 \pm 70$ & $265 \pm 72$ & $\mathrm{mg} \mathrm{O} / \mathrm{L}$ \\
DBO & $169 \pm 24$ & $24 \pm 15$ & $14 \pm 7,3$ & $\mathrm{mg} \mathrm{O} / \mathrm{L}$ \\
COT & $44 \pm 19$ & $21 \pm 8,0$ & $17 \pm 4,0$ & $\mathrm{mg} \mathrm{C} / \mathrm{L}$ \\
\hline
\end{tabular}

\subsection{Potencial de Reúso do Efluente}

Conforme descrito anteriormente, o potencial de reúso do efluente foi avaliado por meio de um balanço de massa utilizando como parâmetro crítico a concentração de Sólidos Dissolvidos Totais (SDT). Para isso, foi determinada a carga de sais incorporada ao sistema por dia com base na concentração de SDT presente na água limpa e no efluente do sistema de tratamento (Tabela 3), resultando em 1,93 kg SDT/dia. A partir deste valor e do equacionamento matemático do sistema de lavagem com a inclusão do reúso, foi possível obter a variação da concentração de SDT na água de reúso em função das porcentagens de recirculação do efluente tratado e de utilização de água limpa para o enxágue final, como mostra a Figura 5.

Com base na Figura 5, observa-se que a concentração de SDT na água de reúso aumenta de forma exponencial caso não seja utilizado o enxágue final com água limpa, tendendo ao infinito quando a porcentagem de recirculação de efluentes tende a $100 \%$. Nesta condição, e considerando o limite de $200 \mathrm{mg} / \mathrm{L}$ proposto pela NBR 13.969 de 1997 para a água de reúso na lavagem de veículos, o potencial máximo de reúso estaria próximo dos $40 \%$. Entretanto, caso seja adotado um procedimento de enxágue final com água limpa, a taxa de reúso pode ser significativamente superior. Nesse sentido, considerando um volume de água para o enxágue final do caminhão equivalente a $30 \%$ do volume necessário para a lavagem, é possível reutilizar $70 \%$ do efluente tratado, sendo a concentração final de SDT na água de reúso de, aproximadamente, $850 \mathrm{mg} / \mathrm{L}$. Com a utilização do procedimento de enxágue com água limpa, mesmo que a concentração de SDT na água de reúso seja superior ao limite proposto na NBR 13.969, o enxágue final com água limpa (SDT $=43 \mathrm{mg} / \mathrm{L}$ ) assegura a eficiência do processo de lavagem e a minimização do potencial de ocorrência de manchas.

Cabe ressaltar que os resultados apresentados neste estudo mostram-se coerentes com aqueles apresentados por Zaneti et al. (2011), embora a metodologia de tratamento de dados utilizada por estes autores tenha sido distinta. Contudo, uma análise detalhada dos dados apresentados pelos mesmos autores permite verificar que as taxas de recirculação de efluentes foram de $45 \%$ e $61 \%$ nas primeira e segunda fase dos estudos respectivamente, tendo sido observada elevação da concentração dos contaminantes na água de reúso.

Outro aspecto relacionado à proposta de reúso deste trabalho diz respeito ao tempo necessário para que a concentração do contaminante de controle atinja o valor de equilíbrio 
determinado pelo balanço de massa, o que depende do volume do tanque de armazenagem da água de reúso. Utilizando-se a Equação 9 com volumes variados do tanque de reúso, foi possível obter o perfil de variação da concentração de SDT com o tempo (Figura 6). Tendo em vista os dados apresentados na Figura 6, verifica-se que quanto maior o volume do tanque de armazenagem da água de reúso, maior será o tempo necessário para que a concentração do contaminante de controle atinja o valor de equilíbrio. Em comparação com os dados de qualidade apresentados no trabalho de Zaneti et al. (2011), observa-se novamente uma concordância no comportamento da variação da concentração de contaminantes com o tempo.

Considerando o potencial de reúso obtido com enxágue utilizando água limpa (70\%), a redução no consumo de água no empreendimento em estudo seria de $5,6 \mathrm{~m}^{3}$ por dia (145,6 $\mathrm{m}^{3}$ por mês), para uma operação do lava-rápido de 6 dias por semana. Uma vez que a água utilizada é proveniente de um poço, a redução de custo na operação do lava-rápido estará associada à redução tanto do consumo de energia para bombeamento quanto do volume de efluentes que deveriam ser lançados à rede. Para o cálculo do consumo de energia de bombeamento, além dos dados sobre a profundidade do poço $(50 \mathrm{~m})$ e eficiência do conjunto moto-bomba (50\%), admite-se que a vazão da bomba do poço é de $5 \mathrm{~m}^{3} / \mathrm{h}$, o que resulta em um tempo mensal de bombeamento de 29,12 horas.

Com base na expressão apresentada por Porto (2006), a potência do conjunto moto-bomba do lava-rápido é de $1,36 \mathrm{~kW}$. Esta potência, associada ao tempo de bombeamento resulta em um consumo mensal de energia igual a 39,6 $\mathrm{kWh}$. Com base na tarifa de energia elétrica praticada pela concessionária (ANEEL, 2016), a redução mensal no consumo de energia elétrica seria de $\mathrm{R} \$ 21,52$. Em relação á tarifa de esgoto, a redução mensal no gasto com a companhia de saneamento seria de, aproximadamente, $\mathrm{R} \$ 2.569,23$, totalizando uma redução no custo operacional do lava-rápido de $\mathrm{R} \$ 2.590,75 /$ mês.

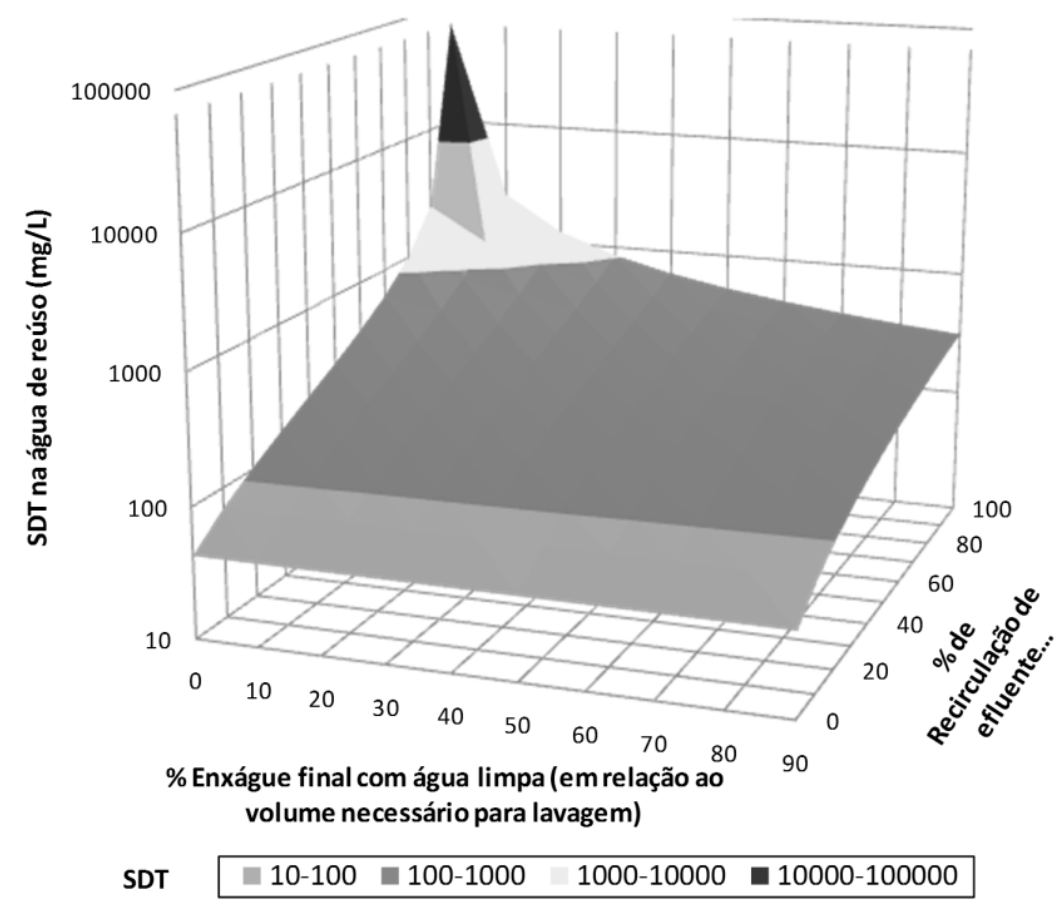

Figura 5. Variação da concentração de SDT na água de reúso em função das porcentagens de recirculação de efluente e de enxágue com água limpa. 


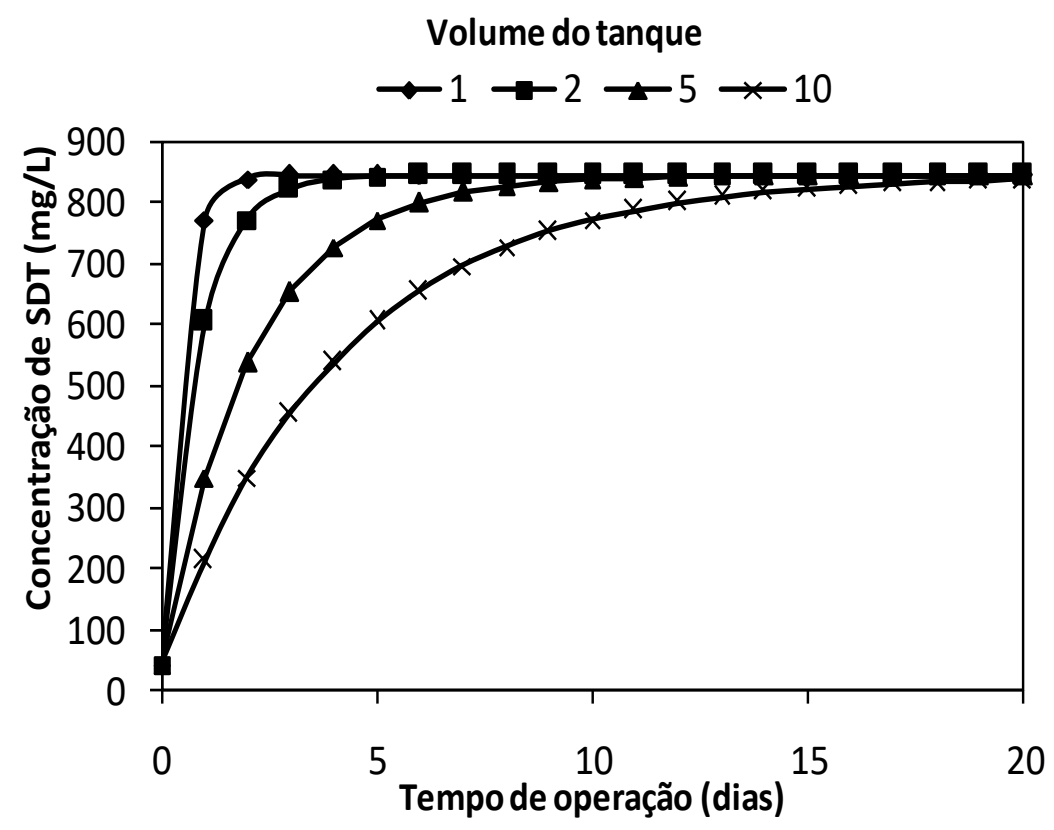

Figura 6. Variação da concentração de SDT na água de reúso em função do tempo e do volume do tanque de reúso.

\section{CONCLUSÕES}

Os resultados obtidos neste estudo demonstraram que o equacionamento adequado de um balanço de massa mostra-se uma ferramenta confiável para avaliação de sistemas de reúso de água, principalmente quando ocorre a elevação da concentração de contaminantes específicos, como os SDT. Foi verificado que o sistema CBR apresenta-se como uma tecnologia viável para o tratamento de efluentes provenientes da lavagem de caminhões, possibilitando reduções significativas de Turbidez, Cor e DBO, além de não promover um aumento na concentração de SDT, o que geralmente ocorre com processos de tratamento físico-químicos. Esta propriedade torna sua operação e manutenção relativamente mais fáceis, quando comparadas a sistemas onde há acúmulo de matéria. A adoção da unidade de tratamento CBR e do enxágue final com água limpa possibilita reutilizar até $70 \%$ do efluente sem que haja problemas com manchas nos veículos devido a elevada concentração de SDT na água de reúso. Observa-se que a lavagem de caminhões é diferente da lavagem de carros de passeio por incluir a lavagem do compartimento de carga. Desta forma, recomenda-se avaliação detalhada da associação do CBR com outros métodos para redução de turbidez e dosagem de auxiliar de filtração, enquanto o controle microbiológico pode ser feito pela cloração do efluente tratado, para assegurar uma qualidade adequada da água de reúso.

\section{AGRADECIMENTOS}

Os autores gostariam de agradecer ao Grupo Teixeira Pinto pelo suporte financeiro.

\section{REFERENCIAS}

AGÊNCIA NACIONAL DE ENERGIA ELÉTRICA - ANEEL (Brasil). Conheça as tarifas da classe de consumo residencial de uma concessionária. 2016. Disponível em http://www2.aneel.gov.br/aplicacoes/tarifaAplicada/index.cfm. Acesso em: 13 set. 2016. 
AGÊNCIA REGULADORA DE SANEAMENTO E ENERGIA DO ESTADO DE SÃO PAULO - ARSESP. Deliberação $n^{\circ} 643$, que dispõe sobre a autorização sobre o reajuste tarifário anual sobre as tarifas vigentes constantes da Deliberação $n^{\circ} 561 / 2015$. Diário Oficial [do] Estado de São Paulo, 12 abr. 2016.

AKUNNA, J. C.; JEFFERIES, C. Performance of family-size sequencing batch reactor and rotating biological contactor units treating sewage at various operating conditions. Water Science Technology, v. 41, p. 97-104, 2000.

AMERICAN PUBLIC HEALTH ASSOCIATION - APHA. Standard methods for the examination of water and wastewater. 21st ed. New York, 2005.

ASSOCIAÇÃO BRASILEIRA DE NORMAS TÉCNICAS - ABNT. NBR 13.969. Tanques sépticos - Unidades de tratamento complementar e disposição final dos efluentes líquidos - Projeto, construção e operação. Rio de Janeiro, 1997.

BOLUARTE I. A. R.; ANDERSEN, M.; PRAMANIK, B. K.; CHANG, C-Y.; BAGSHAW, S.; FARAGO, L. et al. Reuse of car wash wastewater by chemical coagulation and membrane bioreactor treatment processes. International Biodeterioration \& Biodegradation, v. 113, p. 44-48, 2016. http://dx.doi.org/10.1016/j.ibiod.2016.01.017

BHATTI, Z. A.; MAHMOOD, Q.; RAJA, I. A.; MALIK, A. H.; KHAN, M. S., WU, D. Chemical oxidation of carwash industry wastewater as an effort to decrease water pollution. Physics and Chemistry of Earth, v. 36, p. 465-469, 2010. http://dx.doi.org/10.1016/j.pce.2010.03.022

BOUSSU, K.; KINDTS, K.; VANDECASTEELE, C.; VAN DER BRUGGEN, B. Applicability of nanofiltration in the carwash industry. Separation and Purification Technology, v. 54, p. 139-46, 2007. http://dx.doi.org/10.1016/j.seppur.2006.08.024

BRAZIL, B. L. Performance and operation of a rotating biological contactor in a tilapia recirculating aquaculture system. Aquacultural Engineering, v. 34, p. 261-274, 2006. http://dx.doi.org/10.1016/j.aquaeng.2005.06.005

BROWN, C. Water conservation in the professional car wash industry. 1st ed. Washington: International Car Wash Association, 2000.

BROWN C. Water use in the professional car wash industry. 1st ed. Washington: International Car Wash Association, 2002.

ESPIRITO SANTO. Lei N ${ }^{\circ}$ 9.439, de 03 de maio de 2010. Dispõe sobre a obrigatoriedade dos postos de combustíveis, lava-jatos, transportadoras, empresas de ônibus e locadoras de veículos instalarem equipamentos de tratamento e reutilização da água usada na lavagem de veículos. Diário Oficial [do] Estado, 04 maio 2010.

ETCHEPARE, R. G. Integração de processos no tratamento de efluentes de lavagem de veículos para reciclagem de água. 2012. Dissertação (Mestrado) - Programa de PósGraduação em Engenharia de Minas, Metalurgia e Materiais, Universidade Federal do Rio Grande do Sul, Porto Alegre, 2012.

GARCIA-CUERVA, L.; BERGLUND, E. Z.; BINDER, A. R. Public perceptions of water shortages, conservation behaviors, and support for water reuse in the U. S. Resources,

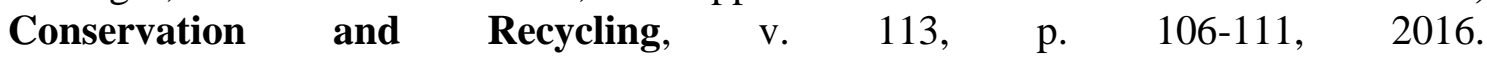
http://dx.doi.org/10.1016/j.resconrec.2016.06.006 
GOIÁS. Lei $N^{\circ}$ 17.128, de 18 de Agosto de 2010. Dispõe sobre a obrigatoriedade de instalação de equipamento para tratamento e reutilização da água utilizada na lavagem de veículos, e de equipamento para reaproveitamento de água das chuvas. Diário Oficial [do] Estado, 14 set. 2010.

GOIÁS. Lei $N^{\circ} 17.582$, de 08 de março de 2012. Altera a Lei ${ }^{\circ} 17.128$, de 18 de agosto de 2010, que dispõe sobre a obrigatoriedade de instalação de equipamento para tratamento e reutilização da água utilizada na lavagem de veículos. Diário Oficial [do] Estado, 15 mar. 2012.

GRADY, C. P. L.; DAIGGER, G. T.; LIM, H. C. Rotating biological contactor. In: LESLIE GRADY JR., C. P.; DAIGGER, G. T.; LOVE, N. G.; FILIPE, C. D. M. Biological wastewater treatment. $2^{\text {nd }}$ Edition. Boca Raton: CRC Press; Taylor \& Francis Group, 1999.

GRIFFIN, P.; FINDLAY, G. E. Process and engineering improvements to rotating biological contactor design. Water Science Technology, v. 41, p. 137-144, 2000.

HAMADA, T.; MIYAZAKI, Y. Reuse of car wash water with a cellulose acetate ultrafiltration membrane aided by flocculation and activated carbon treatments. Desalination, v. 169, p. 257-67, 2004. http://dx.doi.org/10.1016/j.desal.2004.02.089

HASSARD, F.; BIDDLE, J.; CARTMELL, E.; JEFFERSON, J.; TYRREL, S.; STEPHENSON, T. Rotating biological contactors for wastewater treatment - a review. Process Safety and Environmental Protection, v. 94, p. 285-306, 2015. http://dx.doi.org/10.1016/j.psep.2014.07.003

HESPANHOL, I. Potencial de reúso de água no Brasil: agricultura, indústria, municípios e recarga de aquíf́feros. In: MANCUSO, P.; SANTOS, H. Reuso de Água. Barueri: Manole, 2003. p. 37-95.

HESPANHOL, I.; MIERZWA, J. Água na Indústria: uso racional e reúso. São Paulo: Oficina de textos, 2005.

HUYBRECHTS, D.; DE BAERE, P.; VAN ESPEN, L.; WELLENS, B.; DIJKMANS, R. Best Available Techniques for Carwash and Truckwash. BBT Study VITO. Disponível em: http://www.emis.vito.be. Acesso em: set. 2016.

JORDÃO, E. P.; PESSOA, C. A. Tratamento de esgotos domésticos. 6. ed. Rio de Janeiro: ABES, 2011.968 p.

JÖNSSON, C.; JÖNSSON, A. The influence of degreasing agents used at car washes on the performance of ultrafiltration membranes. Desalination, v. 100, p. 115-23, 1995.

LAU, W. J.; ISMAIL, A. F., FIRDAUS, S. Car wash industry in Malysia: Treatment of car wash effluent using ultrafiltration and nanofiltration membranes. Separation and purification Technology, v. 104, p. 26-31, 2013.

http://dx.doi.org/10.1016/j.seppur.2012.11.012

MAY, S. Caracterização, tratamento e reúso de águas cinzas e aproveitamento de águas pluviais em edificações. 2009. Tese (Doutorado) - Universidade de São Paulo, São Paulo, 2009. 
NOWAK, O. Upgrading of wastewater treatment plants equipped with rotating biological contactors to nitrification and P removal. Water Science Technology, v. 41, p. 145$153,2000$.

PANIZZA, G. M.; CERISOLA, G. Applicability of electrochemical methods to carwash wastewaters for reuse. Part 2: electrocoagulation and anodic oxidation integrated process. Journal of Electroanalytical Chemistry, v. 63, p. 236-40, 2010. http://dx.doi.org/10.1016/j.jelechem.2009.11.003

PORTO, R.M. Hidráulica básica. 4. ed. São Paulo: USP, 2006. 519p.

RIO DE JANEIRO. Lei $\mathrm{n}^{\circ}$ 6034, de 08 de setembro de 2011. Dispõe sobre a obrigatoriedade dos postos de combustíveis, lava-rápidos, transportadoras e empresas de ônibus urbanos intermunicipais e interestaduais, localizados no Estado do Rio de Janeiro, a instalarem equipamentos de tratamento e reutilização da água usada na lavagem de veículos. Diário Oficial [do] Estado, 08 set. 2011.

SÃO PAULO (Cidade). Lei no 16.160, de 13 de abril de 2015. Cria o Programa de Reúso de Água em Postos de Serviços e Abastecimento de Veículos e lava-rápidos no Município de São Paulo, e dá outras providências. São Paulo: Secretaria do Governo Municipal, 2015 .

QUEENSLAND WATER COMMISSION (QWC). Standard vehicle washing: fixed commercial premises. Brisbane, 2008a.

QUEENSLAND WATER COMMISSION (QWC). (2008b). Large vehicle washing guideline. Brisbane, 2008b.

ZANETI, R.; ETCHEPARE, R.; RUBIO, J. Car wash wastewater reclamation. Full-scale application and upcoming features. Resources, Conservation and Recycling, v. 55, p. 953-959, 2011. http://dx.doi.org/10.1016/j.resconrec.2011.05.002 